\title{
U.S. Vietnamese parents' HPV vaccine decision-making for their adolescents: an exploration of practice-, provider-, and patient-level influences
}

\author{
Milkie Vu' ${ }^{1}$ ( Robert A. Bednarczyk ${ }^{2,3} \cdot$ Cam Escoffery $^{1,3} \cdot$ Danny Ta $^{4}$. \\ Victoria N. Huynh ${ }^{5}$ Carla J. Berg ${ }^{6,7}$
}

Received: 29 March 2021 / Accepted: 4 November 2021 / Published online: 18 November 2021

(C) The Author(s), under exclusive licence to Springer Science+Business Media, LLC, part of Springer Nature 2021

\begin{abstract}
U.S. Vietnamese have high cervical cancer incidence and low human papillomavirus (HPV) vaccine initiation. Using the P3 model, we explored practice-, provider-, and patient-level determinants of U.S. Vietnamese parents' HPV vaccine decision-making for their adolescents. We conducted a cross-sectional, online survey (04/2020-12/2020) with U.S. Vietnamese parents who had $\geq 1$ adolescent ages 9-18. We assessed HPV vaccination outcomes (initiation, willingness to initiate, completion) and provider recommendation. Modified Poisson regressions were used to identify practice-, provider- and patient-level correlates of outcomes. The sample $(n=408)$ was 44 years old on average; $83 \%$ were female and $85 \%$ had a Bachelor's degree. Around half of
\end{abstract}

Supplementary Information The online version contains supplementary material available at https://doi.org/10.1007/ s10865-021-00265-3.

Milkie Vu

milkie.vu@emory.edu

1 Department of Behavioral, Social, and Health Education Sciences, Rollins School of Public Health, Emory University, Atlanta, GA, USA

2 Hubert Department of Global Health, Rollins School of Public Health, Emory University, Atlanta, GA, USA

3 Winship Cancer Institute, Emory University, Atlanta, GA, USA

4 Neil Hodgson Woodruff School of Nursing, Rollins School of Public Health, Emory University, Atlanta, GA, USA

5 Emory College of Arts and Sciences, Emory University, Atlanta, GA, USA

6 Department of Prevention and Community Health, Milken Institute of Public Health, George Washington University, Washington, DC, USA

7 George Washington Cancer Center, George Washington University, Washington, DC, USA adolescents were female (51\%) and 13-18 year old (54\%). Only 41 and $23 \%$ of parents had initiated and completed the HPV vaccine series for their child, respectively. Initiation was associated with receiving provider recommendation (either low- or high-quality), while willingness to initiate was associated with receiving high-quality recommendation. Both initiation and willingness to initiate was negatively associated with parental perception that their child was too young for a "sexually transmitted infection (STI)-preventing vaccine." Provider recommendation was associated with higher parental U.S. acculturation and the child being older and female. Provider-facing interventions should promote high-quality, age-based, gender-neutral HPV vaccine recommendation. These and population- and individual-facing interventions should recognize the need for additional parental education, particularly related to misconceptions regarding STI prevention.

Keywords Vietnamese $\cdot$ HPV vaccine $\cdot$ Provider recommendation $\cdot$ Sexual activity $\cdot$ Adolescents

\section{Introduction}

U.S. Vietnamese, defined as those living in the U.S. and identifying as Vietnamese, number more than 1.8 million as of 2019, representing the fourth largest Asian group in the U.S. (United States Census Bureau, 2019). Compared to other Asians in the U.S., U.S. Vietnamese have lower English proficiency, median household income, and educational attainment and are more likely to be uninsured and in poverty (United States Census Bureau, 2019). These disadvantages reflect potential barriers to health services utilization and highlight a need to study health behaviors and outcomes in this specific subgroup. 
One important health disparity among this population is cervical cancer rates. Historically, U.S. Vietnamese had the highest cervical cancer rate among all racial and ethnic groups. From 1988 to 1992, the cervical cancer incidence rate (per 100,000) in U.S. Vietnamese was 43.0, compared to 7.5 in non-Hispanic Whites, 13.2 in Blacks, and 16.2 in Hispanic (Miller et al., 1996; Parker et al., 1998; Surveillance Epidemiology \& End Results Program n.d.). The incidence rate has since decreased [possibly due to the implementation of several cervical cancer screening interventions in U.S. Vietnamese communities in the late 1990s and 2000s (Ma et al., 2015; Mock et al., 2007; Scoggins et al., 2010; Taylor et al., 2010) but still remains high. From 2009 to 2011, the cervical cancer incidence rate (per 100,000) was 9.0 in U.S. Vietnamese, versus 6.5 in all Asian Americans and 7.5 in non-Hispanic Whites (Jin et al., 2016). No published data examine periods that are more recent or look at other HPVrelated cancers (e.g., cancers of the vulva, vagina, penis, anus, or oropharynx) among U.S. Vietnamese. As past research has noted, data on HPV-related cancers and other cancers for specific Asian subgroups (including U.S. Vietnamese) are scant due to the tendency to report aggregated instead of disaggregated statistics and the limited availability of data collection tools in Asian languages (Chen, 2005; Holland \& Palaniappan, 2012; Islam et al., 2010; Lee et al., 2019a; Liu et al., 2019; Srinivasan \& Guillermo, 2000; Thompson et al., 2016).

Evidence of high cervical cancer incidence rates points to the circulation of the virus in this population and necessitates solutions to reduce HPV-related cancer burden, particularly through increased HPV vaccine uptake. HPV vaccination is a safe and effective method to prevent HPVrelated cancers, including cervical cancer. To date, limited research has focused on HPV vaccination among U.S. Vietnamese (Duong \& Hopfer, 2020; Gor et al., 2011; Hopfer et al., 2017; Nguyen-Truong et al., 2017; Yi et al., 2013a, 2013b). Findings indicate that U.S. Vietnamese generally have low awareness or knowledge of the vaccine and low HPV vaccine uptake (Duong \& Hopfer, 2020; Gor et al., 2011; Hopfer et al., 2017; Nguyen-Truong et al., 2017; Yi et al., 2013a, 2013b). For example, among 113 Vietnamese women in Houston-based survey, only $14 \%$ reported vaccine initiation (Yi et al., 2013a). Factors related to HPV vaccine intention or uptake among U.S. Vietnamese included English proficiency (Yi et al., 2013a), HPV vaccine knowledge (Yi et al., 2013a), vaccine recommendation from healthcare providers (Hopfer et al., 2017; Yi et al., 2013b), influences from family members (Hopfer et al., 2017; Nguyen-Truong et al., 2017), and school-based education (Hopfer et al., 2017; Nguyen-Truong et al., 2017).

This existing literature regarding HPV vaccination among U.S. Vietnamese has two major limitations. First, existing research has primarily focused on U.S.
Vietnamese young adult females (Duong \& Hopfer, 2020; Gor et al., 2011; Hopfer et al., 2017; Nguyen-Truong et al., 2017; Yi et al., 2013a). Research including U.S. Vietnamese adolescents is limited to only one study assessing mothers' intention to get the HPV vaccine for their daughters (ages 9-26) (Yi et al., 2013b) and no publications have focused on adolescent boys or men. Given that the HPV vaccine is the most effective when administered to adolescents before the age of 15 (Centers for Disease Control \& Prevention, 2020; Pedersen et al., 2007), is currently recommended for both sexes (Centers for Disease Control \& Prevention, 2020), and most likely requires parental consent (Zimet, 2005), research examining U.S. Vietnamese parents' HPV vaccine uptake for both male and female adolescents is critically needed to estimate vaccination coverage and identify modifiable barriers to utilization.

Second, limited research has leveraged a comprehensive health services framework to examine factors at different health system levels influencing HPV vaccine uptake among U.S. Vietnamese. Rather, existing research has concentrated on individual-level factors (e.g., language, vaccine knowledge) (Duong \& Hopfer, 2020; Gor et al., 2011; Hopfer et al., 2017; Nguyen-Truong et al., 2017; Yi et al., 2013a, 2013b) or only looked at a single healthcare system factor-provider recommendation. Research exploring a wide range of factors related to the healthcare environment in which caregivers or patients make decision is needed, particularly because healthcare system-based interventions effectively improve HPV vaccine coverage (Niccolai \& Hansen, 2015; Smulian et al., 2016). One framework that is well-suited to address this specific health behavior and multilevel healthcare factors associated with it is the P3 model (Bednarczyk et al., 2018). The P3 model highlights the roles of practice-, provider-, and patient-level components in the healthcare encounter that influence preventive care behaviors (Bednarczyk et al., 2018) and has been used in previous research examining facilitators of or barriers to HPV vaccination (Vu et al., 2020a, 2020b).

Using the P3 model, we aimed to fill these gaps in the literature by examining U.S. Vietnamese parents' HPV vaccine decision-making for their adolescents of both sexes. Specifically, we examined factors that influence Vietnamese parents' HPV vaccine initiation for their adolescents across three levels: (1) practice (e.g., clinicbased availability of HPV vaccine materials, availability of interpreter or patient navigation services), (2) provider (e.g., provider recommendation quality), and (3) patient (e.g., predisposing, enabling, and reinforcing factors). In addition, we examined P3 factors associated with HPV vaccine completion among initiators and with willingness to initiate HPV vaccine among non-initiators, as well as sociodemographic factors associated with receiving a provider recommendation for the HPV vaccine. 


\section{Methods}

\section{Recruitment and data collection}

The methods employed for the recruitment of this study have been described elsewhere (Vu et al., 2021). Briefly, from April to December 2020, we conducted an online, cross-sectional survey with 408 U.S. Vietnamese parents. We utilized several different venues for recruitment: community-based organizations (CBOs) serving Vietnamese and/or Asians $(\mathrm{n}=60$ CBOs), Vietnamese Students Associations (VSAs, $\mathrm{n}=13$ VSAs), relevant Facebook groups and listserv engaging U.S. Vietnamese $(n=12$ and $n=2$, respectively), the personal network of the first author, and snowball sampling among participants successfully recruited using the aforementioned channels (Sadler et al., 2010). We successfully recruited 68 participants from CBOs and VSAs, 97 from Facebook groups, 4 from listserv, 42 from the first author's network, and 197 from snowball sampling. The overall response rate was $72 \%$ (range: $52-87 \%$ ), as calculated by the American Association for Public Opinion Research's (AAPOR) response rate calculator version 4.1 (American Association for Public Opinion Research, 2020).

Parental eligibility criteria included: (1) self-identified as Vietnamese; (2) having lived in the U.S. for at least 12 months; (3) able to read either Vietnamese or English; and (4) having at least one child ages 9-18 living in the same household. Only one parent per household was allowed to participate. Participants first completed an online eligibility screener (administered via SurveyGizmo). Those eligible were directed to a webpage with a study description and a consent form. Consenting individuals indicated consent via clicking "Yes, I confirm participation in this study" and were directed to the online survey (via SurveyGizmo). The survey took approximately $60 \mathrm{~min}$ to complete. We limited duplicate responses by permitting only one response per IP address. Participants were compensated with a \$30 Amazon gift card.

\section{Measurements}

Survey measures, informed by the literature ( $\mathrm{Vu}$ et al., 2020a), were reviewed by the research team (i.e., experts in HPV vaccine, Vietnamese health, Asian health, and survey research). Participants could choose to take the screener and main survey in either Vietnamese or English. Survey translation was conducted using the Brislin's back-translation method (Brislin, 1970), an iterative translation process involving an independent translation of survey items into Vietnamese and back-translation into English by two different translators, and then reviewed by the first author (who is fully fluent in both languages) and by approximately 10 Vietnamese native speakers to ensure comprehensibility.
Participants were instructed to provide answers about only one child who was between 9 and 18 years old and lived in their home. Those with more than one child in this age range living in their home were instructed to answer these questions considering their oldest child in the age range. Appendix A lists all survey questions.

Outcomes: HPV vaccine initiation, completion, willingness to initiate, and provider recommendation

All outcome measures were adapted from the 2019 National Immunization Survey-Teen (Centers for Disease Control \& Prevention, 2019). HPV vaccine initiation was assessed by asking, "Has your child ever received HPV vaccine shots?" To assess provider recommendation for HPV vaccine, we asked, "Has a doctor or other healthcare professional ever recommended that your child receive HPV vaccine shots?" (response options of yes, no, don't know). For both questions, responses were dichotomized $(0=$ no/don't know, $1=$ yes).

Among those who indicated that they did not initiate the HPV vaccine for their child or did not know whether their child had initiated the HPV vaccine, willingness to initiate HPV vaccine was assessed by asking, "How likely is it that your child will receive HPV vaccine shots in the next 12 months?" Responses were dichotomized $(0=$ not at all/ not very likely/prefer not to answer, $1=$ somewhat/very likely). Among those who indicated their child had initiated the HPV vaccine, we asked, "How many HPV vaccine shots has your child received?" We also asked the age that the child received the first HPV vaccine shot. Per the CDC's Advisory Committee on Immunization Practice's (ACIP) recommendations (Centers for Disease Control \& Prevention, 2020), HPV vaccine completion was defined as having received two doses if the child initiated the HPV vaccine before age 15 and three doses if initiated at 15 or older. Those who indicated they did not know how many vaccine shots their child received were treated as not having completed the series. In addition, we assessed reasons for initiating the HPV vaccine, reasons for not initiating, and reasons for initiating but not completing the vaccine series [adapting previously used measures (Vu et al., 2019a)].

\section{Practice-level correlates}

Newly-created items assessed healthcare practice-level factors relevant to HPV vaccine initiation, including if they had seen materials about the HPV vaccine at their child's primary clinic or had ever used clinic-based patient navigation or language translation/interpretation services (yes or no). Those without a primary clinic were treated as missing data. 


\section{Provider-level correlates}

For those who had received a provider recommendation for the HPV vaccine, we assessed HPV vaccine recommendation quality by asking three questions reflecting different recommendation dimensions (Gilkey et al., 2016a): (1) "How important did the provider say the HPV vaccine was your child?" (response options of $1=$ not at all to $4=$ very important, reflecting "strength"); (2) "Did the healthcare provider tell you that the HPV vaccine prevents cancer?" (response options of $1=$ strongly disagree to $4=$ strongly agree, reflecting "prevention"); and (3) "Did the healthcare provider recommend that your child gets the vaccine that same day?" (response options of $1=$ strongly disagree to $4=$ strongly agree, reflecting "urgency"). Strength was dichotomized as $1=$ very important versus $0=$ other responses; prevention and urgency were dichotomized as $1=$ agree/strongly agree versus $0=$ other responses. We then calculated the summed score of the three items and created two categories: lowquality recommendation (scores of 0-1) and high-quality recommendation (scores of 2-3). Those who did not receive a provider recommendation were grouped into the third category of "no recommendation."

\section{Patient-level correlates}

Guided by the P3 model, we included measurements of predisposing factors (i.e., individual characteristics, beliefs, and values that facilitate or hinder HPV vaccine uptake), enabling factors (i.e., skills, resources, or barriers that facilitate or hinder HPV vaccine uptake), and reinforcing factors (i.e., perceived rewards and feedback received from HPV vaccine uptake) (Bednarczyk et al., 2018; Green \& Kreuter, 2005).

Regarding predisposing factors, we administered the Vaccine Confidence Scale (Gilkey et al., 2016b), which assesses parents' level of general vaccine confidence (i.e., not HPV vaccine-specific) and includes subscales assessing perceived vaccine benefits, vaccine harms, and trust in medical professionals. Participants were asked to indicate their level of agreement $(0=$ strongly disagree to $10=$ strongly agree $)$ to eight statements (e.g., "Vaccines are safe" and "I have a good relationship with my teenager's healthcare provider"). Two items were reverse-coded: "Teenagers receive too many vaccines" and "If I vaccinate my teenager, he/she may have serious side effects." A higher score, derived as an average of item scores, indicates more positive attitudes towards vaccination (Cronbach's alpha $=0.76$ in this sample).

We adapted three items from the Carolina HPV Immunization Attitudes and Beliefs Scale (McRee et al., 2009) (CHIAS) to assess perceived effectiveness of HPV vaccine in preventing genital warts, cervical cancer, and HPV ( $1=$ slightly effective to $5=$ extremely effective). A higher score, derived as an average across items, indicates higher perceived effectiveness of HPV vaccine in preventing diseases (Cronbach's alpha $=0.83$ ).

We used two CHIAS items to assess beliefs about the HPV vaccine and sexual activity. Participants were asked to indicate their level of agreement $(1=$ strongly disagree to $5=$ strongly agree) to: "If a teenager gets the HPV vaccine, he or she may be more likely to have sex" and "My child is too young to get a vaccine for a sexually transmitted infection (STI) like HPV."

Regarding enabling factors, we used a five-item CHIAS subscale to assess perceived barriers related to HPV vaccine access and cost. Participants were asked to indicate their level of agreement ( $1=$ strongly disagree to $5=$ strongly agree) to the statement "I am concerned that the HPV vaccine costs more than I can pay." In addition, they were asked to indicate their perceived level of difficulty ( $1=$ not hard at all to $4=$ very hard) to four statements about ability to find a provider/clinic with affordable vaccine, easy access, available vaccine, and no long wait time for appointments. A higher score, derived as an average of the five item scores, indicates higher perceived barriers in accessing and being able to afford the HPV vaccine (Cronbach's alpha =0.83).

Regarding reinforcing factors, we used a CHIAS item to assess social influence on HPV vaccine uptake. Participants were asked to indicate their level of agreement $(1=$ strongly disagree to $5=$ strongly agree) to: "Other parents in my community are getting their children the HPV vaccine."

\section{Sociodemographic factors}

We assessed: child's age, sex, and country of birth; and parental sex, highest education level, percentage of lifetime in the U.S., and ability to understand medical information in English. In addition, the Asian American Multidimensional Acculturation Scale (Gim Chung et al., 2004) was used to assess parental Vietnamese acculturation and parental American acculturation (Cronbach's alphas of 0.87 and 0.92 , respectively).

\section{Statistical analysis}

All data analyses were conducted in Stata 15.1. Sociodemographic, healthcare practice-, provider-, and patient-level characteristics were summarized using descriptive statistics. Bivariate analyses were conducted using chi-square and independent sample t-tests to examine sociodemographic, healthcare practice-, provider-, and patient-level characteristics in relation to (1) HPV vaccine initiation, (2) HPV vaccine completion among initiators, (3) willingness to initiate HPV vaccine among non-initiators, and (4) receiving a provider recommendation for HPV vaccine. Simple and multivariable modified Poisson regressions with robust error variance were conducted to examine correlates of each 
outcome. We chose this approach instead of logistic regressions because when outcome events are common, odds ratios overestimate risk ratios (Chen et al., 2018; Greenland, 1987; Zou, 2004). In such scenarios, modified Poisson regressions with robust error variance have been shown to provide less biased estimates (Barros \& Hirakata, 2003; Chen et al., 2018; Knol et al., 2012; Zou, 2004). For each outcome, variables with significant associations $(p<0.05)$ in simple regressions were entered into the multivariable regressions. Alpha level was set at 0.05 for all analyses.

\section{Results}

We successfully recruited 408 participants from 36 U.S. states and territories. On average, we recruited 11 participants per state. States with the highest number of participants were California ( $n=117$ or $28.7 \%$ of the sample) and
Texas $(n=61,15.0 \%)$. Six states had the lowest number of participants per state $(n=1,0.2 \%)$.

The overall sample was $83.0 \%$ female with a mean age of 44.81. A majority of parents had at least a Bachelor's degree $(85.1 \%)$. More than half of the parents $(54.4 \%)$ had adolescents ages 13 to 18 . The distribution of child's sex was roughly similar (50.7\% female); so was child's country of birth (48.5\% born in the U.S.). On average, parents had spent around a third of their life in the U.S, but only $37.5 \%$ of parents reported that they could understand medical information in English well (Table 1).

Only $37.1 \%$ of parents reported seeing materials about HPV vaccine at the child's primary clinic. A small number reported using patient navigation services $(6.7 \%)$ or using language interpretation or translation services $(11.1 \%)$. More than half reported receiving no provider recommendation for HPV vaccine, $13.5 \%$ reported receiving a low-quality recommendation, and approximately a third reported receiving a high-quality recommendation.

Table 1 Sociodemographic characteristics in relation to HPV vaccine initiation

\begin{tabular}{|c|c|c|c|c|}
\hline \multirow[t]{3}{*}{ Variable } & Total & Vaccine initiation-yes & $\begin{array}{l}\text { Vaccine initiation-no/ } \\
\text { do not know }\end{array}$ & \multirow[t]{3}{*}{$p$ Value } \\
\hline & $\mathrm{N}=408$ & $N=166$ & $\mathrm{~N}=242$ & \\
\hline & $\mathrm{N}(\%)$ or $\mathrm{M}(\mathrm{SD})$ & $\mathrm{N}(\%)$ or $\mathrm{M}(\mathrm{SD})$ & $\mathrm{N}(\%)$ or $\mathrm{M}(\mathrm{SD})$ & \\
\hline \multicolumn{5}{|l|}{ Child's age } \\
\hline $9-10$ & $89(21.8 \%)$ & $9(5.4 \%)$ & $80(33.1 \%)$ & \multirow[t]{3}{*}{$<0.001$} \\
\hline $11-12$ & $97(23.8 \%)$ & $42(25.3 \%)$ & $55(22.7 \%)$ & \\
\hline $13-18$ & $222(54.4 \%)$ & $115(69.3 \%)$ & $107(44.2 \%)$ & \\
\hline \multicolumn{5}{|l|}{ Child's sex ${ }^{a}(n=406)$} \\
\hline Male & $200(49.3 \%)$ & $64(38.8 \%)$ & $136(56.4 \%)$ & \multirow[t]{2}{*}{$<0.001$} \\
\hline Female & $206(50.7 \%)$ & $101(61.2 \%)$ & $105(43.6 \%)$ & \\
\hline \multicolumn{5}{|l|}{ Child's country of birth } \\
\hline Born in the U.S & $198(48.5 \%)$ & $81(48.8 \%)$ & $117(48.4 \%)$ & \multirow[t]{2}{*}{0.93} \\
\hline Born outside of the U.S & $210(51.5 \%)$ & $85(51.2 \%)$ & $125(51.7 \%)$ & \\
\hline \multicolumn{5}{|l|}{ Parent's sex ${ }^{a}(n=405)$} \\
\hline Male & $69(17.0 \%)$ & $23(13.9 \%)$ & $46(19.2 \%)$ & \multirow[t]{2}{*}{0.17} \\
\hline Female & $336(83.0 \%)$ & $142(86.1 \%)$ & $194(80.8 \%)$ & \\
\hline \multicolumn{5}{|l|}{ Parent's highest education level } \\
\hline Less than a Bachelor's degree & $61(15.0 \%)$ & $26(15.7 \%)$ & $35(14.5 \%)$ & \multirow[t]{3}{*}{0.02} \\
\hline Bachelor's degree & $157(38.5 \%)$ & $51(30.7 \%)$ & $106(43.8 \%)$ & \\
\hline Master's degree or doctoral degree & $190(46.6 \%)$ & $89(53.6 \%)$ & $101(41.7 \%)$ & \\
\hline Parent's percentage of lifetime in the U.S & $33.72(25.38)$ & $35.14(26.28)$ & $32.75(24.76)$ & 0.35 \\
\hline \multicolumn{5}{|c|}{ Parent's ability to understand medical information in English } \\
\hline Not at all to somewhat easy & $255(62.5 \%)$ & $95(57.2 \%)$ & $160(66.1 \%)$ & \multirow[t]{2}{*}{0.07} \\
\hline Very to extremely easy & $153(37.5 \%)$ & $71(42.8 \%)$ & $82(33.9 \%)$ & \\
\hline Parental Vietnamese acculturation ${ }^{b}(n=407)$ & $4.15(0.60)$ & $4.14(0.63)$ & $4.16(0.58)$ & 0.74 \\
\hline Parental American acculturation & $2.88(0.82)$ & $3.00(0.79)$ & $2.80(0.84)$ & 0.02 \\
\hline
\end{tabular}

aThose who chose "Other" or "Prefer not to answer" were coded as having missing data

${ }^{\mathrm{b}}$ Those who indicated that their heritage culture was not Vietnamese $(\mathrm{n}=1)$ were coded as having missing data 
Approximately a quarter of parents agreed that their child was too young for an STI-preventing vaccine like the HPV vaccine. In addition, approximately a third agreed that other parents in their community are getting their children the HPV vaccine (Table 2).

\section{HPV vaccine initiation}

The proportion of those who initiated the HPV vaccine for their child was $40.7 \%$ of the entire sample $(n=166 / 408)$, $27.4 \%$ of those with adolescents ages $9-12(n=51 / 186)$, and $51.8 \%$ of those with adolescents ages 13-18

Table 2 Healthcare practice-, provider-, and patient-level characteristics in relation to HPV vaccine initiation

\begin{tabular}{|c|c|c|c|c|}
\hline \multirow[t]{3}{*}{ Variable } & Total & Vaccine initiation-yes & $\begin{array}{l}\text { Vaccine initiation- } \\
\text { no/do not know }\end{array}$ & \multirow[t]{3}{*}{$p$ Value } \\
\hline & $\mathrm{N}=408$ & $N=166$ & $\mathrm{~N}=242$ & \\
\hline & $\mathrm{N}(\%)$ or $\mathrm{M}(\mathrm{SD})$ & $\mathrm{N}(\%)$ or $\mathrm{M}(\mathrm{SD})$ & $\mathrm{N}(\%)$ or $\mathrm{M}(\mathrm{SD})$ & \\
\hline \multicolumn{5}{|l|}{ Practice-level factors } \\
\hline \multicolumn{5}{|c|}{ Parent seen materials about HPV vaccine at primary $\operatorname{clinic}^{\mathrm{a}}(\mathrm{n}=404)$} \\
\hline No & $141(34.9 \%)$ & $37(22.4 \%)$ & $104(43.5 \%)$ & \multirow[t]{3}{*}{$<0.001$} \\
\hline Yes & $150(37.1 \%)$ & $89(53.9 \%)$ & $61(25.5 \%)$ & \\
\hline Do not know or remember & $113(28.0 \%)$ & $39(23.6 \%)$ & $74(31.0 \%)$ & \\
\hline \multicolumn{5}{|l|}{ Parent used patient navigation services ${ }^{\mathrm{a}}(\mathrm{n}=404)$} \\
\hline No & $320(79.2 \%)$ & $128(77.6 \%)$ & $192(80.3 \%)$ & \multirow[t]{3}{*}{0.80} \\
\hline Yes & $27(6.7 \%)$ & $12(7.3 \%)$ & $15(6.3 \%)$ & \\
\hline Do not know & $57(14.1 \%)$ & $25(15.2 \%)$ & $32(13.4 \%)$ & \\
\hline \multicolumn{5}{|c|}{ Parent used language interpretation or translation services ${ }^{a}(n=404)$} \\
\hline No/Do not know ${ }^{b}$ & $359(88.9 \%)$ & $152(92.1 \%)$ & $207(86.6 \%)$ & \multirow[t]{2}{*}{0.08} \\
\hline Yes & $45(11.1 \%)$ & $13(7.9 \%)$ & $32(13.4 \%)$ & \\
\hline \multicolumn{5}{|l|}{ Provider-level factors } \\
\hline \multicolumn{5}{|l|}{ Provider's HPV vaccine recommendation quality } \\
\hline No recommendation ${ }^{\mathrm{b}}$ & $222(54.4 \%)$ & $13(7.8 \%)$ & $209(86.4 \%)$ & \multirow[t]{3}{*}{$<0.001$} \\
\hline Low-quality recommendation & $55(13.5 \%)$ & $39(23.5 \%)$ & $16(6.6 \%)$ & \\
\hline High-quality recommendation & $131(32.1 \%)$ & $114(68.7 \%)$ & $17(7.0 \%)$ & \\
\hline \multicolumn{5}{|l|}{ Patient-level factors } \\
\hline Vaccine confidence scale ${ }^{c}$ & $7.52(1.45)$ & $7.85(1.35)$ & $7.29(1.48)$ & $<0.001$ \\
\hline Perceived effectiveness of HPV vaccine ${ }^{d}$ & $3.18(0.85)$ & $3.34(0.91)$ & $3.07(0.80)$ & 0.002 \\
\hline \multicolumn{5}{|c|}{ Child more likely to have sex after HPV vaccine initiation } \\
\hline Disagree/strongly disagree & $297(72.8 \%)$ & $139(83.7 \%)$ & $158(65.3 \%)$ & \multirow[t]{3}{*}{$<0.001$} \\
\hline Agree/strongly agree & $33(8.1 \%)$ & $6(3.6 \%)$ & $27(11.2 \%)$ & \\
\hline Do not know & $78(19.1 \%)$ & $21(12.7 \%)$ & $57(23.6 \%)$ & \\
\hline \multicolumn{5}{|c|}{ Child too young for a STI-prevention vaccine like the HPV vaccine } \\
\hline Disagree/strongly disagree & $248(60.8 \%)$ & $143(86.1 \%)$ & $105(43.4 \%)$ & \multirow[t]{3}{*}{$<0.001$} \\
\hline Agree/strongly agree & $103(25.3 \%)$ & $9(5.4 \%)$ & $94(38.8 \%)$ & \\
\hline Do not know & $57(14.0 \%)$ & $14(8.4 \%)$ & $43(17.8 \%)$ & \\
\hline Perceived barriers of HPV vaccine access and costs ${ }^{\mathrm{e}}$ & $1.75(0.69)$ & $1.51(0.56)$ & $1.92(0.73)$ & $<0.001$ \\
\hline \multicolumn{5}{|c|}{ Other parents in the community are getting children HPV vaccine } \\
\hline Disagree/strongly disagree & $56(13.7 \%)$ & $21(12.7 \%)$ & $35(14.5 \%)$ & \multirow[t]{3}{*}{$<0.001$} \\
\hline Agree/strongly agree & $143(35.1 \%)$ & $83(50.0 \%)$ & $60(24.8 \%)$ & \\
\hline Do not know & $209(51.2 \%)$ & $62(37.4 \%)$ & $147(60.7 \%)$ & \\
\hline
\end{tabular}

${ }^{a}$ Those who responded that their child did not have a primary clinic $(n=4)$ were coded as having missing data

b The categories of "No" and "Do not know" were combined because responses of "Do not know" were less than $10 \%$ of total responses

${ }^{\mathrm{c}}$ Range 2.38-10, mean 7.52, standard deviation 1.45, a higher score indicates more positive attitudes towards vaccination

${ }^{\mathrm{d}}$ Range 1-5, mean 3.18, standard deviation 0.85 , a higher score indicates higher perceived effectiveness of HPV vaccine in preventing diseases

${ }^{\mathrm{e}}$ Range $1-4.2$, mean 1.75, standard deviation 0.69 , a higher score indicates higher perceived barriers in accessing and being able to afford the HPV vaccine 
$(n=115 / 222)$. The most common reasons for vaccine initiation included doctor's recommendation $(83.7 \%)$, wanting to protect child from diseases (49.4\%), ensuring the child gets all recommended vaccines $(33.1 \%)$, and thinking that HPV-related diseases are serious $(24.7 \%)$ (Table 3).

In bivariate analyses (Model 1, Table 4), HPV vaccine initiation was associated with seeing clinic-based materials about HPV vaccine and provider recommendation (both low and high quality). In addition, HPV vaccine initiation was associated with multiple patient-level vaccine-specific factors: higher vaccine confidence, higher perceived effectiveness of HPV vaccine, lower perception that the child is more likely to have sex after HPV vaccine initiation, lower perception that the child was too young for an STI-preventing vaccine like the HPV vaccine, lower perceived barriers related to HPV vaccine access and cost, and higher perception that other parents in the community are getting the HPV vaccine. HPV vaccine initiation was also associated with child being older and female as well as higher parental education, ability to understand medical information in English, and American acculturation.

In multivariable regression (Model 2, Table 4), compared to those receiving no provider recommendation, those receiving a low-quality recommendation (adjusted relative risks or aRR $=9.08 ; 95 \% \mathrm{CI}=5.04-16.36$ ) and high-quality recommendation $(\mathrm{aRR}=10.60 ; 95 \%$ $\mathrm{CI}=5.96-18.85)$ were more likely to have initiated the HPV vaccine for their child. Higher perception that the child was too young for an STI-preventing vaccine like the HPV vaccine was associated with lower likelihood of vaccine initiation $(\mathrm{aRR}=0.73 ; 95 \% \mathrm{CI}=0.63-0.84)$.

\section{HPV vaccine completion}

The proportion of those who completed the HPV vaccine series was $23.3 \%$ of the entire sample $(n=95 / 408), 9.1 \%$ of those with adolescents ages $9-12(n=17 / 186), 35.1 \%$ of those with adolescents ages $13-18(n=78 / 222)$, and $57.2 \%$ of those who initiated the vaccine $(n=95 / 166)$. The most common reasons for having initiated but not completed the series (Table 3 ) included child starting the series too recent to finish $(40.8 \%)$ and not knowing child needed more than one dose $(15.5 \%)$.

In bivariate analyses (Model 1, Table 5), factors associated with HPV vaccine completion included higher vaccine confidence, lower perception that the child was too young for an STI-preventing vaccine like the HPV vaccine, and older child's age. In multivariable regression (Model 2, Table 5), HPV vaccine completion was associated with older child's age $(\mathrm{aRR}=1.10,95 \% \mathrm{CI}=1.04-1.17)$.

\section{Willingness to initiate HPV vaccine}

Of those who had not initiated the HPV vaccine for their child $(n=242), 45 \%$ reported that they were somewhat or very likely to initiate the vaccine in the next 12 months. The most common reasons for not having initiated HPV vaccine (Table 3) included a lack of doctor's recommendation $(62.8 \%)$, doctor's indication that child could get vaccinated at an older age $(14.1 \%)$, not thinking that child needs the vaccine because of a lack of sexual activity (13.2\%), no school requirements $(12.0 \%)$, and not knowing any HPVrelated diseases $(10.7 \%)$.

In bivariate analyses (Model 1, Table 5), factors associated with willingness to initiate HPV vaccine included receiving a high-quality recommendation, higher vaccine

Table 3 Most common reasons underlying HPV vaccine initiation, non-initiation, or initiation but non-completion

\begin{tabular}{ll}
\hline Reasons & $\mathrm{N}(\%)$ \\
\hline Most common reasons for initiating HPV vaccine (total $\mathrm{n}=166)$ & $139(83.7 \%)$ \\
A doctor recommended it for my child & $82(49.4 \%)$ \\
I want to protect my child from diseases & $55(33.1 \%)$ \\
I get my child all recommended vaccines & $41(24.7 \%)$ \\
I think HPV-related diseases are serious & $29(40.8 \%)$ \\
Most common reasons for having initiated but not completed HPV vaccine series (total $\mathrm{n}=71)$ & $11(15.5 \%)$ \\
My child started the series too recently to finish & $152(62.8 \%)$ \\
I didn't know my child needed more than 1 dose & $34(14.1 \%)$ \\
Most common reasons for not having initiated HPV vaccine (total n=242) & $32(13.2 \%)$ \\
Doctor never recommended HPV vaccine for my child & $29(12.0 \%)$ \\
Doctor indicated that my child can get vaccinated at an older age & $26(10.7 \%)$ \\
My child is not sexually active, so I do not think the vaccine is necessary & \\
My child's school does not require vaccination, so I do not think the vaccine is necessary & \\
I do not know about any HPV-related disease &
\end{tabular}


Table 4 Healthcare practice-, provider-, and patient-level predictors of HPV vaccine initiation

\begin{tabular}{|c|c|c|}
\hline Variable & $\begin{array}{l}\text { Model } 1 \text { (unadjusted) } \\
\text { cRR }(95 \% \text { CI) }\end{array}$ & $\begin{array}{l}\text { Model } 2 \text { (adjusted) } \\
\text { aRR }(95 \% \text { CI) }\end{array}$ \\
\hline \multicolumn{3}{|l|}{ Practice-level factors } \\
\hline \multicolumn{3}{|l|}{ Parent seen materials about HPV vaccine at primary clinic } \\
\hline No/Do not know & Reference & Reference \\
\hline Yes & $1.98(1.57-2.50)^{* * *}$ & $1.16(0.98-1.37)$ \\
\hline \multicolumn{3}{|l|}{ Provider-level factors } \\
\hline \multicolumn{3}{|l|}{ Provider's HPV vaccine recommendation quality } \\
\hline No recommendation & Reference & Reference \\
\hline Low-quality recommendation & $12.11(6.95-21.09) * * *$ & $9.08(5.04-16.36)^{* * *}$ \\
\hline High-quality recommendation & $14.86(8.73-25.30)^{* * *}$ & $10.60(5.96-18.85)^{* * *}$ \\
\hline \multicolumn{3}{|l|}{ Patient-level factors } \\
\hline Vaccine confidence scale & $1.18(1.09-1.28)^{* * *}$ & $0.98(0.91-1.06)$ \\
\hline Perceived effectiveness of HPV vaccine & $1.24(1.08-1.43)^{* *}$ & $0.98(0.89-1.08)$ \\
\hline Child is more likely to have sex after HPV vaccine initiation & $0.77(0.66-0.89)^{* * *}$ & $1.04(0.94-1.15$ \\
\hline Child is too young for a STI-prevention vaccine like HPV vaccine & $0.58(0.51-0.65)^{* * *}$ & $0.73(0.63-0.84)^{* * *}$ \\
\hline Perceived barriers related to HPV vaccine access and cost & $0.52(0.41-0.67)^{* * *}$ & $0.86(0.72-1.01)$ \\
\hline Other parents in the community are getting HPV vaccine & $1.37(1.18-1.58)^{* * *}$ & $1.05(0.96-1.15)$ \\
\hline \multicolumn{3}{|l|}{ Sociodemographic characteristics } \\
\hline Child's age & $1.14(1.10-1.19)^{* * *}$ & $1.01(0.98-1.04)$ \\
\hline \multicolumn{3}{|l|}{ Child's sex } \\
\hline Male & Reference & Reference \\
\hline Female & $1.53(1.20-1.96)^{* *}$ & $1.07(0.92-1.25)$ \\
\hline \multicolumn{3}{|l|}{ Parent's highest education level } \\
\hline Bachelor's degree or less & Reference & Reference \\
\hline Master's degree or doctoral degree & $1.33(1.05-1.68)^{*}$ & $1.12(0.96-1.31)$ \\
\hline Parent's ability to understand medical information in English & $1.13(1.00-1.27)^{*}$ & $0.97(0.88-1.07)$ \\
\hline Parental American acculturation & $1.20(1.03-1.39)^{*}$ & $0.93(0.81-1.08)$ \\
\hline
\end{tabular}

Simple and multivariable modified Poisson regressions with robust error variance were conducted to examine correlates of the outcome $* p<0.05$

$* * p<0.01$

$* * * p<0.001$

confidence, higher perceived effectiveness of HPV vaccine, lower perception that the child is more likely to have sex after HPV vaccine initiation, and lower perception that the child was too young for an STI-preventing vaccine like the HPV vaccine. In multivariable regression (Model 2, Table 5), compared to those receiving no provider recommendation, those receiving high-quality recommendation were more likely to be willing to initiate the HPV vaccine for their child ( $\mathrm{aRR}=1.57,95 \%$ $\mathrm{CI}=1.15-2.14)$. Higher perception that the child was too young for an STI-preventing vaccine like the HPV vaccine was associated with lower likelihoods of willingness to initiate the vaccine $(\mathrm{aRR}=0.86 ; 95 \% \mathrm{CI}=0.77-0.98$ ).

\section{Provider recommendation for HPV vaccine}

In the sample, $45.6 \%$ indicated that they had ever received provider recommendation and $32.1 \%$ indicated receiving a high-quality recommendation. Among all parents who received provider recommendation $(n=186)$, only $22.0 \%$ indicated that the provider recommended for the child to start the HPV vaccine at an age of 12 or younger (not shown in tables). In bivariate analyses (not shown in tables), sociodemographic correlates of receiving provider recommendation were child being older and female, higher parental percentage of lifetime in the U.S., ability to understand medical information in English, and American acculturation. 
Table 5 Healthcare practice-, provider, and patient-level predictors of HPV vaccine completion \& willingness to initiate HPV vaccine

\begin{tabular}{|c|c|c|}
\hline Variable & $\begin{array}{l}\text { Model } 1 \text { (unadjusted) } \\
\text { cRR }(95 \% \mathrm{CI})\end{array}$ & $\begin{array}{l}\text { Model } 2 \text { (adjusted) } \\
\operatorname{aRR}(95 \% \mathrm{CI})\end{array}$ \\
\hline \multicolumn{3}{|c|}{ Outcome: vaccine completion (among those who had initiated the vaccine for their children, $n=166$ ) } \\
\hline Vaccine confidence scale & $1.12(1.01-1.24)^{*}$ & $1.05(0.94-1.17)$ \\
\hline Child is too young for a STI-prevention vaccine like HPV vaccine & $0.79(0.65-0.98)^{*}$ & $0.84(0.68-1.05)$ \\
\hline Child's age & $1.11(1.05-1.18)^{* * *}$ & $1.10(1.04-1.17)^{* *}$ \\
\hline \multicolumn{3}{|c|}{ Outcome: willingness to initiate HPV vaccine (among those with unvaccinated children, $n=242$ ) } \\
\hline \multicolumn{3}{|l|}{ Provider's HPV vaccine recommendation quality } \\
\hline No recommendation & Reference & Reference \\
\hline Low-quality recommendation & $1.22(0.72-2.04)$ & $1.22(0.72-2.08)$ \\
\hline High-quality recommendation & $1.57(1.07-2.32)^{*}$ & $1.57(1.15-2.14)^{* *}$ \\
\hline Vaccine confidence scale & $1.14(1.03-1.25)^{* *}$ & $1.05(0.95-1.17)$ \\
\hline Perceived effectiveness of HPV vaccine & $1.29(1.08-1.54)^{* *}$ & $1.16(0.98-1.38)$ \\
\hline Child is more likely to have sex after vaccine initiation & $0.82(0.70-0.96)^{*}$ & $0.91(0.77-1.08)$ \\
\hline Child is too young for a STI-prevention vaccine like HPV vaccine & $0.82(0.73-0.92)^{* *}$ & $0.86(0.77-0.98)^{*}$ \\
\hline
\end{tabular}

Simple and multivariable modified Poisson regressions with robust error variance were conducted to examine correlates of each outcome $* p<0.05$

$* * p<0.01$

$* * * p<0.001$

In multivariable regression (not shown in tables), receiving provider recommendation was associated with the child being older $(\mathrm{aRR}=1.15,95 \% \mathrm{CI}=1.11-1.20)$ and female $(\mathrm{aRR}=1.35,95 \% \mathrm{CI}=1.10-1.65)$, and higher parental American acculturation $(\mathrm{aRR}=1.27,95 \% \mathrm{CI}=1.09-1.48)$. Of those who had received provider recommendation, we did not find any significant associations between sociodemographic characteristics and receiving low-quality versus high-quality recommendations (not shown in tables).

\section{Discussion}

Our study represents the first effort to examine healthcare practice-, provider-, and patient-level determinants of U.S. Vietnamese parents' HPV vaccine decision-making for their adolescent children. We found that only $41 \%$ had initiated and $23 \%$ had completed the vaccine series for their child; further, $46 \%$ received provider recommendation for HPV vaccine. Vaccine initiation was associated with receiving provider recommendation for vaccination (either low- or high-quality), while willingness to initiate the vaccine was associated with receiving a high-quality recommendation (which occurred among 32\% of participants). In addition, both vaccine initiation and willingness to initiate the vaccine was negatively associated with parental perception that their child was too young for an STI-preventing vaccine.

Among adolescent ages 13 to 18 in our sample, the prevalence of HPV vaccine initiation and completion was 52 and $35 \%$, respectively. In contrast, the 2020 NIS-Teen, which surveyed vaccination coverage of U.S. adolescents ages 13-17, estimated that 75 and 59\% of all adolescents and 77 and $61 \%$ non-Hispanic Asian adolescents had initiated and completed the HPV vaccine series, respectively (Pingali et al., 2021). Moreover, the on-time initiation rate among those ages 9-12 in our sample was $27 \%$, in contrast to the nationwide rate of $63 \%$ in 2018 (Chido-Amajuoyi et al., 2021). These stark disparities necessitate strategies and interventions to address low adolescent HPV vaccination coverage among U.S. Vietnamese. Furthermore, interventions should aim to address barriers to not only HPV vaccine initiation but also vaccine completion, particularly given that $16 \%$ of parents who had initiated the vaccine did not know that their child needed more than one dose.

The strong positive association between provider recommendation and parents' HPV initiation for their child, as documented in our study, has also been reported in general populations (Newman et al., 2018), immigrant populations (Kim \& LeClaire, 2017), Asian populations (Dela Cruz et al., 2018; Vu et al., 2020a), and U.S. Vietnamese parents of daughters ages 9-26 (Yi et al., 2013b). However, unlike most previous studies that only measured the presence or absence of provider recommendation, we assessed several aspects of recommendation (e.g., quality, recommended age, subgroups more likely to receive recommendation). While Vietnamese parents receiving high-quality recommendations were more likely to be willing to initiate the vaccine for their child in the next 12 months, no association was found among those receiving low-quality recommendations. A similar result was reported in a study with U.S. parents, 
where parents who had initially refused HPV vaccination but received a high-quality recommendation were more likely to get their child vaccinated during a later visit (i.e. secondary acceptance) (Kornides et al., 2018). This study also found that low-quality recommendations did not have an impact on secondary acceptance (Kornides et al., 2018).

Less than a quarter of all Vietnamese parents who received provider recommendation reported that their providers recommended an age of 12 or younger for their child to start the HPV vaccine. This finding may reflect documented practices where providers based their recommendations on perceptions of adolescents' sexual risks or access to care rather than the ages in ACIP's guidelines (Henrikson et al., 2016). Additionally, Vietnamese parents of sons were less likely to have received a provider recommendation compared to parents of daughters, a pattern also noted in previous research with general U.S. populations (Beavis et al., 2018). Moreover, through using validated measurements for acculturation, we found that Vietnamese parents with higher U.S. acculturation were more likely to receive a provider recommendation, independent of their ability to understand medical information in English or duration in the U.S. It is possible that those with higher U.S. acculturation may place a higher emphasis on preventive care (Geltman et al., 2014) and thus more likely to take their child to a doctor's visit, thereby creating more opportunities to receive a provider recommendation. This association should be further explored in Vietnamese populations and other immigrant populations.

Taken together, these findings indicate the need for actions to ensure high-quality and age-based provider recommendation for HPV vaccine for U.S Vietnamese parents of both male and female adolescents. Dimensions of high-quality recommendations can include emphasizing the importance and cancer prevention values of the vaccine, recommending same-day vaccination, delivering recommendations to all adolescents by age 12 , and recommending the HPV vaccine at the same time and in the same way as other adolescent vaccines (Gilkey \& McRee, 2016). The literature has documented several effective provider-focused interventions for general U.S. populations that utilized educational presentations, audit and feedback, and training in communication approach with parents (Leung et al., 2019), though we are not aware of research implementing such interventions in healthcare systems serving U.S. Vietnamese or Asian communities. For example, a multi-component intervention in Colorado implemented webinar and in-person communication training for providers regarding the use of the "presumptive announcement" approach to open the HPV vaccine conversation and motivational interviewing strategies for vaccine hesitant parents (Dempsey et al., 2018; Reno et al., 2018, 2019). Providers reported that the communication training was one of the most important components of the intervention, and adolescents in the intervention practices were significantly more likely to initiate and complete the HPV vaccine series compared to those in the control practices (Dempsey et al., 2018). Another multi-component intervention in Ohio provides both communication training to all providers and individualized audits to providers who missed an opportunity to vaccinate a patient against HPV (Krantz et al., 2018). The intervention resulted in more consistently-occurring vaccination during visits and increased vaccine initiation and completion rates (Krantz et al., 2018).

A quarter of parents in the sample believed that their child was too young for an STI-preventing vaccine. This perception was negatively associated with HPV vaccine initiation and willingness to initiate the vaccine. In addition, of parents who had not initiated the HPV vaccine, $13 \%$ cited their child's lack of sexual activity as a reason for non-initiation. A few studies have reported similar concerns in Korean, Cambodian, and Haitian immigrant populations (Do et al., 2009; Lee et al., 2019b; Stephens \& Thomas, 2013). The 2016 NIS-Teen data indicated that between 9 and $10 \%$ of U.S. parents reported their child's lack of sexual activity as a primary reason for why they would not get their child vaccinated in the next 12 months (Beavis et al., 2018). Educational programs and interventions should address this perception by emphasizing that HPV vaccine is the most effective when administered prior to sexual debut (Adams et al., 2007). Moreover, sexual activity is not the only possible source of exposure to HPV, as there are non-sexual and nonpenetrative sources of HPV transmission (Liu et al., 2016).

It should be noted that our study took place from April to December 2020 during the COVID-19 pandemic. HPV vaccine ordering and coverage dropped substantially in 2020 (Daniels et al., 2021; Gilkey et al., 2020), possibly due to a number of challenges such as decreased capacity of primary clinics, limited interactions between providers and patients, and parents' delay of preventive care visits based on fears of COVID-19 exposure (Gilkey et al., 2020). These barriers could have led to reduced opportunities for HPV vaccination for adolescents in our study, particularly those who became eligible for vaccination during the period of this research. Recent efforts to improve HPV vaccine uptake have emphasized strategies such as co-administration of HPV vaccine with COVID-19 vaccines for adolescents (Committee on Infectious Diseases at the American Academy of Pediatrics, 2021).

\section{Strengths and limitations}

Strengths of our study include the recruitment of U.S. Vietnamese parents from diverse sources (e.g., community-based organizations, Facebook groups) and the use of a rigorous translation process. We assessed determinants of HPV vaccine decision-making using comprehensive, validated 
measurements (e.g., multiple dimensions of provider recommendation or of acculturation). Nevertheless, our survey is cross-sectional, which limits interpretations of temporal relationships between variables. We also relied on parents' self-reports of HPV vaccination status, which is subjected to inaccuracy (Vu et al., 2019b). Additionally, we assessed the vaccination status of the oldest child, which can be subjected to recall bias. While we did not find any association between practice-level factors and HPV vaccine outcomes, this result could be due to the fact that we captured practicelevel factors from parents' perspectives. Future studies could consider exploring the perspectives of clinic staff or using ethnographic research to further understand practice-level influences on HPV vaccine decision-making. Other confounding variables, such as the length or quality of the relationship between patients and providers, can also be considered. Given our focus on parents' perspectives, we were not able to measure additional provider-level correlates that may be important, such as provider's attitudes, beliefs, or practices regarding HPV vaccination. Additionally, we measured parents' perceptions of provider recommendation quality, which may not always match with provider's perceptions.

The generalizability of our findings may have been limited given that the majority of parents were college-educated. It is possible that those who have higher education were more likely to participate in health research (Ashford et al., 2020; Würtzen et al., 2013). We note, however, that recent systematic reviews in general and Asian American populations have not identified a definite relationship between education and HPV vaccine uptake (Newman et al., 2018; Radisic et al., 2017; Rodriguez et al., 2020; Vu et al., 2020a). Moreover, almost all parents (99.3\%) in our study reported that their child had health insurance. Since having health insurance has been shown to be associated with higher HPV vaccine uptake (Kessels et al., 2012; Newman et al., 2018), the observed level of vaccine uptake in our study may have been lower if more Vietnamese parents with uninsured children had been included.

\section{Conclusions}

U.S. Vietnamese parents reported low HPV vaccine initiation and completion for their adolescents. Future providerfacing interventions should promote high-quality, age-based, gender-neutral HPV vaccine recommendation. These and population- and individual-facing interventions should recognize the need for additional parental education, particularly related to misconceptions regarding STI prevention.

Authors' contribution MV is responsible for conceptualizing the study, designing the survey, overseeing data collection, performing data analysis, and drafting and editing the manuscript. RAB, CE, and CJB all contributed to survey designing, data analysis, and manuscript editing. DT and VHN contributed to data collection, data analysis, and manuscript editing. All authors have reviewed the submitted manuscript and approve the manuscript for submission.

Funding This work is supported by the American Psychological Foundation 2019 Visionary Grant and the American Association for Cancer Education 2019 Grant in Research, Education, Advocacy, and Direct Service (READS). Our data collection receives support from the Center for AIDS Research at Emory University (P30AI050409). Dr. Vu is supported by the National Cancer Institute (F31CA243220), a 2020-2021 PEO Scholar Award, and the 2020-2021 Student Fellowship in Patient Engagement from the Society of Public Health Education. Dr. Berg is supported by the US National Cancer Institute (R01CA215155-01A1; R01CA179422-01; R01CA239178-01A1), the US Fogarty International Center/National Institutes of Health (1R01TW010664-01), and the US National Institute on Environmental Health Science/Fogarty International Center (D43ES030927-01). Dr. Bednarczyk is supported in part by the US National Cancer Institute (1R37CA234119-01).

\section{Declarations}

Conflict of interest The authors declare that they have no conflicts of interest.

Ethics approval for research involving human participants We received approval for research involving human participants from the Institutional Review Board at Emory University (IRB 00111688). All procedures performed in studies involving human participants were in accordance with the ethical standards of the institutional and/or national research committee and with the 1964 Helsinki Declaration and its later amendments or comparable ethical standards.

Informed consent/consent to participate Electronic/online informed consent was provided by all participants.

Animal studies No animal studies were carried out by the authors.

\section{References}

Adams, M., Jasani, B., \& Fiander, A. (2007). Human papilloma virus (HPV) prophylactic vaccination: Challenges for public health and implications for screening. Vaccine, 25, 3007-3013. https://doi. org/10.1016/j.vaccine.2007.01.016

American Association for Public Opinion Research. (2020). American Association for Public Opinion Research-Response Rate Calculator 4.1. Retrieved December 23, 2020, from https://www.aapor. org/Education-Resources/For-Researchers/Poll-Survey-FAQ/ Response-Rates-An-Overview.aspx

Ashford, M. T., Eichenbaum, J., Williams, T., Camacho, M. R., Fockler, J., Ulbricht, A., et al. (2020). Effects of sex, race, ethnicity, and education on online aging research participation. Alzheimer's \& Dementia: Translational Research \& Clinical Interventions. https://doi.org/10.1002/trc2.12028

Barros, A. J., \& Hirakata, V. N. (2003). Alternatives for logistic regression in cross-sectional studies: An empirical comparison of models that directly estimate the prevalence ratio. $B M C$ 
Medical Research Methodology, 3, 21. https://doi.org/10.1186/ 1471-2288-3-21

Beavis, A., Krakow, M., Levinson, K., \& Rositch, A. F. (2018). Reasons for lack of HPV vaccine initiation in NIS-teen over time: shifting the focus from gender and sexuality to necessity and safety. Journal of Adolescent Health, 63, 652-656. https://doi. org/10.1016/j.jadohealth.2018.06.024

Bednarczyk, R. A., Chamberlain, A., Mathewson, K., Salmon, D. A., \& Omer, S. B. (2018). Practice-, provider-, and patient-level interventions to improve preventive care: development of the P3 model. Preventive Medicine Reports, 11, 131-138. https://doi.org/ 10.1016/j.pmedr.2018.06.009

Brislin, R. W. (1970). Back-translation for cross-cultural research. Journal of Cross-Cultural Psychology, 1, 185-216. https://doi. org/10.1177/135910457000100301

Centers for Disease Control and Prevention. (2019). NIS-teen hard copy questionnaire. https://www.cdc.gov/vaccines/imz-managers/ nis/downloads/NIS-Teen-Questionnaire-Q4-2019-508.pdf

Centers for Disease Control and Prevention. (2020). HPV vaccine recommendations. Retrieved December 18, 2020, from https://www. cdc.gov/vaccines/vpd/hpv/hcp/recommendations.html

Chen, M. S. (2005). Cancer health disparities among Asian Americans. Cancer, 104(S12), 2895-2902. https://doi.org/10.1002/cncr.21501

Chen, W., Qian, L., Shi, J., \& Franklin, M. (2018). Comparing performance between log-binomial and robust Poisson regression models for estimating risk ratios under model misspecification. BMC Medical Research Methodology, 18, 63. https://doi.org/10. 1186/s12874-018-0519-5

Chido-Amajuoyi, O. G., Talluri, R., Wonodi, C., \& Shete, S. (2021). Trends in HPV vaccination initiation and completion within ages 9-12 Years: 2008-2018. Pediatrics, 147, e2020012765. https:// doi.org/10.1542/peds.2020-012765

Committee on Infectious Diseases at the American Academy of Pediatrics. (2021). COVID-19 vaccines in children and adolescents. Pediatrics, 148, e2021052336. https://doi.org/10.1542/peds. 2021-052336

Daniels, V., Saxena, K., Roberts, C., Kothari, S., Corman, S., Yao, L., \& Niccolai, L. (2021). Impact of reduced human papillomavirus vaccination coverage rates due to COVID-19 in the United States: A model based analysis. Vaccine, 39, 2731-2735. https://doi.org/ 10.1016/j.vaccine. 2021.04 .003

Dela Cruz, M. R. I., Braun, K. L., Tsark, J. A. U., Albright, C. L., \& Chen, J. J. (2018). HPV vaccination prevalence, parental barriers and motivators to vaccinating children in Hawai'i. Ethnicity and Health. https://doi.org/10.1080/13557858.2018.1473556

Dempsey, A. F., Pyrznawoski, J., Lockhart, S., Barnard, J., Campagna, E. J., Garrett, K., et al. (2018). Effect of a health care professional communication training intervention on adolescent human papillomavirus vaccination. JAMA Pediatrics, 172, e180016. https:// doi.org/10.1001/jamapediatrics.2018.0016

Do, H., Seng, P., Talbot, J., Acorda, E., Coronado, G. D., \& Taylor, V. M. (2009). HPV vaccine knowledge and beliefs among Cambodian American parents and community leaders. Asian Pacific Journal of Cancer Prevention : APJCP, 10, 339-344.

Duong, H. T., \& Hopfer, S. (2020). "Let's Chat": Process evaluation of an intergenerational group chat intervention to increase cancer prevention screening among Vietnamese American families. Translational Behavioral Medicine. https://doi.org/10.1093/tbm/ ibaa120

Geltman, P. L., Hunter Adams, J., Penrose, K. L., Cochran, J., Rybin, D., Doros, G., et al. (2014). Health literacy, acculturation, and the use of preventive oral health care by Somali Refugees living in Massachusetts. Journal of Immigrant and Minority Health, 16, 622-630. https://doi.org/10.1007/s10903-013-9846-0

Gilkey, M. B., Bednarczyk, R. A., Gerend, M. A., Kornides, M. L., Perkins, R. B., Saslow, D., et al. (2020). Getting human papillomavirus vaccination back on track: Protecting our national investment in human papillomavirus vaccination in the COVID-19 era. Journal of Adolescent Health, 67, 633-634. https://doi.org/ 10.1016/j.jadohealth.2020.08.013

Gilkey, M. B., Calo, W. A., Moss, J. L., Shah, P. D., Marciniak, M. W., \& Brewer, N. T. (2016a). Provider communication and HPV vaccination: The impact of recommendation quality. Vaccine, 34, 1187-1192. https://doi.org/10.1016/j.vaccine.2016.01.023

Gilkey, M. B., \& McRee, A. L. (2016). Provider communication about HPV vaccination: A systematic review. Human Vaccines and Immunotherapeutics. https://doi.org/10.1080/21645515.2015. 1129090

Gilkey, M. B., Reiter, P. L., Magnus, B. E., McRee, A.-L., Dempsey, A. F., \& Brewer, N. T. (2016b). Validation of the vaccination confidence scale: A brief measure to identify parents at risk for refusing adolescent vaccines. Academic Pediatrics, 16, 42-49. https://doi.org/10.1016/j.acap.2015.06.007

Gim Chung, R. H., Kim, B. S. K., \& Abreu, J. M. (2004). Asian American multidimensional acculturation scale: Development, factor analysis, reliability, and validity. Cultural Diversity and Ethnic Minority Psychology, 10, 66-80. https://doi.org/10.1037/10999809.10.1.66

Gor, B. J., Chilton, J. A., Camingue, P. T., \& Hajek, R. A. (2011). Young Asian Americans' knowledge and perceptions of cervical cancer and the human papillomavirus. Journal of Immigrant and Minority Health, 13, 81-86. https://doi.org/10.1007/ s10903-010-9343-7

Green, L. W., \& Kreuter, M. W. (2005). Health promotion planning: An educational and ecological approach. McGraw-Hill.

Greenland, S. (1987). Interpretation and choice of effect measures in epidemiologic analyses. American Journal of Epidemiology, 125, 761-768. https://doi.org/10.1093/oxfordjournals.aje.a114593

Henrikson, N. B., Tuzzio, L., Gilkey, M. B., \& McRee, A.-L. (2016). "You're never really off time": Healthcare providers' interpretations of optimal timing for HPV vaccination. Preventive Medicine Reports, 4, 94-97. https://doi.org/10.1016/j.pmedr.2016.05.002

Holland, A. T., \& Palaniappan, L. P. (2012). Problems with the collection and interpretation of Asian-American health data: Omission, aggregation, and extrapolation. Annals of Epidemiology, 22, 397-405. https://doi.org/10.1016/j.annepidem.2012.04.001

Hopfer, S., Garcia, S., Duong, H. T., Russo, J. A., \& Tanjasiri, S. P. (2017). A narrative engagement framework to understand HPV vaccination among Latina and Vietnamese women in a planned parenthood setting. Health Education and Behavior, 44, 738-747. https://doi.org/10.1177/1090198117728761

Islam, N. S., Khan, S., Kwon, S., Jang, D., Ro, M., \& Trinh-Shevrin, C. (2010). Methodological issues in the collection, analysis, and reporting of granular data in Asian American populations: Historical challenges and potential solutions. Journal of Health Care for the Poor and Underserved, 21, 1354-1381. https://muse.jhu. edu/article/400774

Jin, H., Pinheiro, P. S., Xu, J., \& Amei, A. (2016). Cancer incidence among Asian American populations in the United States, 2009 2011. International Journal of Cancer, 138, 2136-2145. https:// doi.org/10.1002/ijc.29958

Kessels, S. J. M., Marshall, H. S., Watson, M., Braunack-Mayer, A. J., Reuzel, R., \& Tooher, R. L. (2012). Factors associated with HPV vaccine uptake in teenage girls: A systematic review. Vaccine, 30 , 3546-3556. https://doi.org/10.1016/j.vaccine.2012.03.063

Kim, K., \& LeClaire, A.-R. (2017). A systematic review of factors influencing human papillomavirus vaccination among immigrant parents in the United States. Health Care for Women International. https://doi.org/10.1080/07399332.2017.1404064

Knol, M. J., Le Cessie, S., Algra, A., Vandenbroucke, J. P., \& Groenwold, R. H. H. (2012). Overestimation of risk ratios by odds ratios in trials and cohort studies: Alternatives to logistic 
regression. Canadian Medical Association Journal, 184, 895899. https://doi.org/10.1503/cmaj.101715

Kornides, M. L., McRee, A.-L., \& Gilkey, M. B. (2018). Parents Who decline HPV vaccination: Who later accepts and why? Academic Pediatrics, 18, S37-S43. https://doi.org/10.1016/j. acap.2017.06.008

Krantz, L., Ollberding, N. J., Beck, A. F., \& Carol Burkhardt, M. (2018). Increasing HPV vaccination coverage through providerbased interventions. Clinical Pediatrics, 57, 319-326. https:// doi.org/10.1177/0009922817722014

Lee, A. W., Navajas, E. E., \& Liu, L. (2019a). Clear differences in ovarian cancer incidence and trends by ethnicity among Asian Americans. Cancer Epidemiology, 61, 142-149. https://doi.org/ 10.1016/j.canep.2019.06.005

Lee, Y.-M., Mondragón, E., Jeong, Y. M., Lee, H. H., Aquino, E., \& Shim, K. (2019b). Exploring the need of HPV education programs in Korean American Communities. Journal of Community Health Nursing, 36, 19-30. https://doi.org/10.1080/07370 016.2018 .1555312

Leung, S. O. A., Akinwunmi, B., Elias, K. M., \& Feldman, S. (2019). Educating healthcare providers to increase Human Papillomavirus (HPV) vaccination rates: A qualitative systematic review. Vaccine: X, 3, 100037. https://doi.org/10.1016/j.jvacx.2019. 100037

Liu, L., Zhang, J., Deapen, D., Stern, M. C., Sipin, A., Pandol, S. J., \& Setiawan, V. W. (2019). Differences in pancreatic cancer incidence rates and temporal trends across Asian subpopulations in California (1988-2015). Pancreas, 48, 931-933. https://doi.org/ 10.1097/MPA.0000000000001337

Liu, Z., Rashid, T., \& Nyitray, A. G. (2016). Penises not required: A systematic review of the potential for human papillomavirus horizontal transmission that is non-sexual or does not include penile penetration. Sexual Health. https://doi.org/10.1071/SH15089

Ma, G. X., Fang, C., Tan, Y., Feng, Z., Ge, S., \& Nguyen, C. (2015). Increasing cervical cancer screening among Vietnamese Americans: A community-based intervention trial. Journal of Health Care for the Poor and Underserved, 26, 36-52. https://doi.org/ 10.1353/hpu.2015.0064

McRee, A.-L., Brewer, N. T., Reiter, P. L., Gottlieb, S. L., \& Smith, J. S. (2009). The Carolina HPV immunization attitudes and beliefs scale (CHIAS): Scale development and associations with intentions to vaccinate. Sexually Transmitted Diseases, 37, 234-239. https://doi.org/10.1097/OLQ.0b013e3181c37e15

Miller, B. A., Kolonel, L. N., Bernstein, L., Young, J. L., Swanson, G. M., West, D. (1996). Racial/ethnic patterns of cancer in the United States 1988-1992. In B. A. Miller (Ed.) NIH Pub. N. National Cancer Institute.

Mock, J., McPhee, S. J., Nguyen, T., Wong, C., Doan, H., Lai, K. Q., et al. (2007). Effective lay health worker outreach and mediabased education for promoting cervical cancer screening among Vietnamese American women. American Journal of Public Health, 97, 1693-1700. https://doi.org/10.2105/AJPH.2006. 086470

Newman, P. A., Logie, C. H., Lacombe-Duncan, A., Baiden, P., Tepjan, S., Rubincam, C., et al. (2018). Parents' uptake of human papillomavirus vaccines for their children: A systematic review and meta-analysis of observational studies. British Medical Journal Open, 8, e019206. https://doi.org/10.1136/bmjopen-2017-019206

Nguyen-Truong, C. K. Y., Nguyen, K. Q. V., Nguyen, T. H., Le, T. V., Truong, A. M., Rodela, K., \& Allan, R. (2017). Vietnamese American women's beliefs and perceptions on cervical cancer, cervical cancer screening, and cancer prevention vaccines: A community-based participatory study. Asian/pacific Island Nursing Journal, 2, 133-142. https://doi.org/10.9741/23736658.1067

Niccolai, L. M., \& Hansen, C. E. (2015). Practice- and communitybased interventions to increase human papillomavirus vaccine coverage. JAMA Pediatrics, 169, 686. https://doi.org/10.1001/ jamapediatrics.2015.0310

Parker, S. L., Davis, K. J., Wingo, P. A., Ries, L. A., \& Heath, C. W. (1998). Cancer statistics by race and ethnicity. CA: a Cancer Journal for Clinicians, 48, 31-48. https://doi.org/10.3322/canjc lin.48.1.31

Pedersen, C., Petaja, T., Strauss, G., Rumke, H. C., Poder, A., Richardus, J. H., et al. (2007). Immunization of early adolescent females with human papillomavirus type 16 and $18 \mathrm{~L} 1$ virus-like particle vaccine containing AS04 adjuvant. Journal of Adolescent Health, 40, 564-571. https://doi.org/10.1016/j.jadohealth.2007.02.015

Pingali, C., Yankey, D., Elam-Evans, L. D., Markowitz, L. E., Williams, C. L., Fredua, B., McNamara, L., \& Stokley, S. (2021). National, regional, state, and selected local area vaccination coverage among adolescents aged 13-17 years United States, 2020. MMWR. Morbidity and Mortality Weekly Report, 70, 1183-1190. https://doi.org/10.15585/mmwr.mm7035a1

Radisic, G., Chapman, J., Flight, I., \& Wilson, C. (2017). Factors associated with parents' attitudes to the HPV vaccination of their adolescent sons: A systematic review. Preventive Medicine. https:// doi.org/10.1016/j.ypmed.2016.11.019

Reno, J. E., O'Leary, S. T., Pyrzanowski, J., Lockhart, S., Thomas, J., \& Dempsey, A. F. (2018). Evaluation of the implementation of a multicomponent intervention to improve health care provider communication about human papillomavirus vaccination. Academic Pediatrics, 18, 882-888. https://doi.org/10.1016/j.acap. 2018.08.004

Reno, J. E., Thomas, J., Pyrzanowski, J., Lockhart, S., O’Leary, S. T., Campagna, E. J., \& Dempsey, A. F. (2019). Examining strategies for improving healthcare providers' communication about adolescent HPV vaccination: Evaluation of secondary outcomes in a randomized controlled trial. Human Vaccines \& Immunotherapeutics, 15, 1592-1598. https://doi.org/10.1080/21645515.2018.1547607

Rodriguez, S. A., Mullen, P. D., Lopez, D. M., Savas, L. S., \& Fernández, M. E. (2020). Factors associated with adolescent HPV vaccination in the U.S.: A systematic review of reviews and multilevel framework to inform intervention development. Preventive Medicine, 131, 105968. https://doi.org/10.1016/j.ypmed.2019.105968

Sadler, G. R., Lee, H.-C., Lim, R.S.-H., \& Fullerton, J. (2010). Research Article: Recruitment of hard-to-reach population subgroups via adaptations of the snowball sampling strategy. Nursing \& Health Sciences, 12, 369-374. https://doi.org/10.1111/j.14422018.2010.00541.x

Scoggins, J. F., Ramsey, S. D., Jackson, J. C., \& Taylor, V. M. (2010). Cost effectiveness of a program to promote screening for cervical cancer in the Vietnamese-American population. Asian Pacific Journal of Cancer Prevention : APJCP, 11, 717-22. http://www. ncbi.nlm.nih.gov/pubmed/21039042

Smulian, E. A., Mitchell, K. R., \& Stokley, S. (2016). Interventions to increase HPV vaccination coverage: A systematic review. Human Vaccines \& Immunotherapeutics, 12, 1566-1588. https://doi.org/ $10.1080 / 21645515.2015 .1125055$

Srinivasan, S., \& Guillermo, T. (2000). Toward improved health: Disaggregating Asian American and Native Hawaiian/Pacific Islander data. American Journal of Public Health, 90, 1731-1734. https:// doi.org/10.2105/ajph.90.11.1731

Stephens, D. P., \& Thomas, T. L. (2013). Cultural values influencing immigrant Haitian mothers' attitudes toward human papillomavirus vaccination for daughters. Journal of Black Psychology, 39, 156-168. https://doi.org/10.1177/0095798412461807

Surveillance Epidemiology and End Results Program. (n.d.). Cancer Stat Facts: Cervical Cancer. https://seer.cancer.gov/statfacts/html/ cervix.html

Taylor, V. M., Jackson, J. C., Yasui, Y., Nguyen, T. T., Woodall, E., Acorda, E., et al. (2010). Evaluation of a cervical cancer control intervention using lay health workers for Vietnamese American 
Women. American Journal of Public Health, 100, 1924-1929. https://doi.org/10.2105/AJPH.2009.190348

Thompson, C. A., Gomez, S. L., Hastings, K. G., Kapphahn, K., Yu, P., Shariff-Marco, S., et al. (2016). The burden of cancer in Asian Americans: A report of national mortality trends by Asian ethnicity. Cancer Epidemiology Biomarkers \& Prevention, 25, 13711382. https://doi.org/10.1158/1055-9965.EPI-16-0167

United States Census Bureau. (2019). 2019 American community survey 1 -year estimates total population. Retrieved September 27, 2020, from http://data.census.gov

Vu, M., Bednarczyk, R. A., Escoffery, C., Getachew, B., \& Berg, C. J. (2019a). Human papillomavirus vaccination among diverse college students in the state of Georgia: Who receives recommendation, who initiates and what are the reasons? Health Education Research. https://doi.org/10.1093/her/cyz014

Vu, M., Berg, C. J., Escoffery, C., Jang, H. M., Nguyen, T. T., Travis, L., \& Bednarczyk, R. A. (2020a). A systematic review of practice-, provider-, and patient-level determinants impacting AsianAmericans' human papillomavirus vaccine intention and uptake. Vaccine, 38, 6388-6401. https://doi.org/10.1016/j.vaccine.2020. 07.059

Vu, M., Huynh, V. N., Bednarczyk, R. A., Escoffery, C., Ta, D., Nguyen, T. T., \& Berg, C. J. (2021). Experience and lessons learned from multi-modal internet-based recruitment of U.S. Vietnamese into research. PLoS ONE, 16, e256074. https://doi. org/10.1371/journal.pone.0256074

Vu, M., King, A. R., Jang, H. M., \& Bednarczyk, R. A. (2020b). Practice-, provider- and patient-level facilitators of and barriers to HPV vaccine promotion and uptake in Georgia: A qualitative study of healthcare providers' perspectives. Health Education Research, 35, 512-523. https://doi.org/10.1093/her/cyaa026
Vu, M., Luu, M., Haardörfer, R., Berg, C. J., Escoffery, C., \& Bednarczyk, R. A. (2019b). A multilevel analysis of factors influencing the inaccuracy of parental reports of adolescent HPV vaccination status. Vaccine. https://doi.org/10.1016/j.vaccine.2018.12.032

Würtzen, H., Dalton, S. O., Andersen, K. K., Elsass, P., Flyger, H. L., Sumbundu, A., \& Johansen, C. (2013). Who participates in a randomized trial of mindfulness-based stress reduction (MBSR) after breast cancer? A study of factors associated with enrollment among Danish breast cancer patients. Psycho-Oncology, 22, 1180-1185. https://doi.org/10.1002/pon.3094

Yi, J. K., Anderson, K. O., Le, Y. C., Escobar-Chaves, S. L., \& ReyesGibby, C. C. (2013a). English proficiency, knowledge, and receipt of HPV vaccine in Vietnamese-American Women. Journal of Community Health, 38, 805-811. https://doi.org/10.1007/ s10900-013-9680-2

Yi, J. K., Lackey, S. C., Zahn, M. P., Castaneda, J., \& Hwang, J. P. (2013b). Human papillomavirus knowledge and awareness among Vietnamese mothers. Journal of Community Health. https://doi. org/10.1007/s10900-013-9709-6

Zimet, G. D. (2005). Improving adolescent health: Focus on HPV vaccine acceptance. Journal of Adolescent Health, 37, S17-S23. https://doi.org/10.1016/j.jadohealth.2005.09.010

Zou, G. (2004). A modified Poisson regression approach to prospective studies with binary data. American Journal of Epidemiology, 159, 702-706. https://doi.org/10.1093/aje/kwh090

Publisher's Note Springer Nature remains neutral with regard to jurisdictional claims in published maps and institutional affiliations. 